\title{
3D PIC-MCC simulations of discharge inception around a sharp anode in nitrogen/oxygen mixtures
}

\author{
Jannis Teunissen ${ }^{1}$ and Ute Ebert ${ }^{1,2}$ \\ ${ }^{1}$ Centrum Wiskunde \& Informatica, PO Box 94079, 1090 GB Amsterdam, The Netherlands \\ 2 Department of Applied Physics, Eindhoven University of Technology, PO Box 513, \\ 5600 MB Eindhoven, The Netherlands \\ E-mail: jannis@ teunissen.net
}

Received 8 March 2016

Accepted for publication 10 May 2016

Published 14 June 2016

\begin{abstract}
We investigate how photoionization, electron avalanches and space charge affect the inception of nanosecond pulsed discharges. Simulations are performed with a 3D PIC-MCC (particlein-cell, Monte Carlo collision) model with adaptive mesh refinement for the field solver. This model, whose source code is available online, is described in the first part of the paper. Then we present simulation results in a needle-to-plane geometry, using different nitrogen/ oxygen mixtures at atmospheric pressure. In these mixtures non-local photoionization is important for the discharge growth. The typical length scale for this process depends on the oxygen concentration. With $0.2 \%$ oxygen the discharges grow quite irregularly, due to the limited supply of free electrons around them. With $2 \%$ or more oxygen the development is much smoother. An almost spherical ionized region can form around the electrode tip, which increases in size with the electrode voltage. Eventually this inception cloud destabilizes into streamer channels. In our simulations, discharge velocities are almost independent of the oxygen concentration. We discuss the physical mechanisms behind these phenomena and compare our simulations with experimental observations.
\end{abstract}

Keywords: discharge, nanosecond, inception, 3D, PIC-MCC, photoionization

(Some figures may appear in colour only in the online journal)

\section{Introduction}

Pulsed streamer discharges are widely used in plasma and high-voltage technology, in applications such as pollution control [1], ozone generation [2], ignition [3] and plasma medicine $[4,5]$. The energy efficiency of corona streamer treatment can be enhanced by faster voltage rise times, because thicker and more ionized streamer channels are created [6,7]. Current technology has enabled the generation of voltage pulses with a duration of one to a few nanoseconds [8]. In chapter 7 of [9] it was observed that pulses of about a nanosecond are too short for the formation of separate streamer channels. Pulses with nanosecond rise times can generate large transient electric fields. In such fields the energy distribution of free electrons is far from equilibrium, and most energy is converted in a few fast electron-neutral processes, while the gas overall stays cold.

During the first stage of a pulsed air discharge a nearly spherical ionized region can form around a pointed electrode. This discharge mode has been termed 'diffuse discharge' by Tardiveau et al [10,11], although they realized that space charge effects were involved. Briels et al [12] had earlier introduced the term 'inception cloud' for the same phenomenon. In earlier work [13] Briels et al argued that the inception cloud's size and degree of ionization are determined by the voltage rise time, and that the properties of later emerging streamers are determined by the destabilization and break-up of the cloud.

In this paper, we investigate the inception of nanosecond pulsed discharges using 3D particle-in-cell simulations in a 
needle-to-plane electrode geometry. We consider positive discharges, i.e. the needle electrode has a positive voltage. Because such discharges grow opposite to the electron drift velocity, they require a source of electrons ahead of them. Our simulations are performed in nitrogen/oxygen mixtures, in which photoionization is typically the most important nonlocal source of electrons $[14,15]$. Because the amount of photoionization produced depends on the oxygen concentration [16], we can study the effect of photoionization by varying the amount of oxygen. We perform simulations under qualitatively similar conditions as in the experimental investigations of for example $[15,17,18]$. In air, we observe inception clouds and their break-up into streamers, whereas the smoothing effect of photo-ionization is missing with a lower oxygen content, and 'feather'-like structures $[19,20]$ are formed rather than inception clouds.

The paper is divided in two major parts. In section 2, the components of the 3D PIC model and the implementation of the electrode are described in detail. This model was used before to study discharge inception in homogeneous fields [21]. In section 3, simulation results are presented for different nitrogen-oxygen mixtures and electrode voltages between 3.5 and $5 \mathrm{kV}$, all at atmospheric pressure. We discuss how the discharge morphology depends on the oxygen concentration and the electrode voltage, and compare with experimental observations.

\section{Simulation model}

We use a PIC-MCC (particle-in-cell, Monte Carlo collision $[22,23])$ model, in which electrons are tracked as particles. Ions are included as densities, and neutral gas molecules are present as a homogeneous background that electrons randomly collide with, as described in section 2.2. Here we consider time scales up to about $10 \mathrm{~ns}$, so the ions are assumed to be immobile. One of the main advantages of using a 3D PIC-MCC model is that we can study how stochastic fluctuations affect the three-dimensional discharge evolution. In [24] it was shown that such density fluctuations are be important for streamer branching, and in [25] their effect on negative streamers was observed.

The model presented below was used before in [21, 26-29]. Here it is for the first time described in more detail, in particular the implementation of the particle mover, electric field solver and electrode. Note that all source code is available at the homepage of our group [30].

\subsection{Particle mover}

We assume to work under electrostatic conditions without an external magnetic field, so that the acceleration of a particle is given by $\mathbf{a}=(\mathrm{q} / \mathrm{m}) \mathbf{E}$, where $\mathbf{E}$ is the electric field and $q / \mathrm{m}$ represents charge over mass. Positions and velocities are updated in time with the Velocity Verlet scheme [31], defined as:

$$
\mathbf{x}(t+\delta)=\mathbf{x}(t)+\delta \mathbf{v}(t)+\frac{1}{2} \delta^{2} \mathbf{a}(t)
$$

$$
\mathbf{v}(t+\delta)=\mathbf{v}(t)+\frac{1}{2} \delta[\mathbf{a}(t)+\mathbf{a}(t+\delta)] .
$$

We do not directly implement the above scheme, however. Because of the Monte Carlo collisions described below, electrons often have to be advanced over a partial time step. Suppose that $\delta$ is subdivided into two steps, such that $\delta=\delta_{1}+\delta_{2}$. The first update is then performed as

$$
\begin{aligned}
& \mathbf{x}\left(t+\delta_{1}\right)=\mathbf{x}(t)+\delta_{1} \mathbf{v}(t)+\frac{1}{2} \delta_{1}^{2} \mathbf{a}(t), \\
& \mathbf{v}\left(t+\delta_{1}\right)=\mathbf{v}(t)+\delta_{1} \mathbf{a}(t),
\end{aligned}
$$

and the second one as

$$
\begin{aligned}
& \mathbf{x}\left(t+\delta_{1}+\delta_{2}\right)=\mathbf{x}\left(t+\delta_{1}\right)+\delta_{2} \mathbf{v}\left(t+\delta_{1}\right)+\frac{1}{2} \delta_{2}^{2} \mathbf{a}(t), \\
& \mathbf{v}\left(t+\delta_{1}+\delta_{2}\right)=\mathbf{v}\left(t+\delta_{1}\right)+\delta_{2} \mathbf{a}(t) .
\end{aligned}
$$

Substitution of variables shows that this is equal to

$$
\begin{aligned}
& \mathbf{x}\left(t+\delta_{1}+\delta_{2}\right)=\mathbf{x}(t)+\left(\delta_{1}+\delta_{2}\right) \mathbf{v}(t)+\frac{1}{2}\left(\delta_{1}+\delta_{2}\right)^{2} \mathbf{a}(t), \\
& \mathbf{v}\left(t+\delta_{1}+\delta_{2}\right)=\mathbf{v}(t)+\left(\delta_{1}+\delta_{2}\right) \mathbf{a}(t),
\end{aligned}
$$

which can directly be generalized to an arbitrary number of partial time steps $\delta=\delta_{1}+\delta_{2}+\cdots+\delta_{n}$. In order to recover the original scheme of (B.2), $\frac{1}{2} \delta[\mathbf{a}(t+\delta)-\mathbf{a}(t)]$ is added to $\mathbf{v}\left(t+\delta_{1}+\delta_{2}+\cdots+\delta_{n}\right)$. An important property of the above procedure is that the occurrence of null collisions (see the next section) does not alter the position and velocity of particles.

\subsection{Collisions}

The discharges that we consider will be weakly ionized, having a degree of ionization of about $10^{-5}$ up to $10^{-4}$. Under such conditions, electron-neutral collisions dominate, so that electron-electron and electron-ion collisions can be neglected. We include the following electron-neutral collisions: elastic scattering, inelastic scattering (due to e.g. electronic excitations), electron impact ionization, and electron attachment, using the $\mathrm{N}_{2}$ and $\mathrm{O}_{2}$ cross sections from the Siglo database [32] on LXcat [33].

The $N_{\text {coll }}$ energy-dependent cross sections $\sigma_{i}$ are converted to collision rates $R_{i}=N \sigma_{i} v$, where $N$ is the density of scattering targets and $v$ the particle velocity. When a particle collides depends on its total collision rate, given by $\sum_{i} R_{i}$. This rate depends on the particle's velocity/energy, and therefore also on time, which greatly complicates the sampling of collision times. The null-collision method [34] resolves this by adding a null collision (with no effect) that makes the total collision rate constant. In other words, the null collision rate is given by $R_{0}=R_{\max }-\sum_{i} R_{i}$, where $R_{\max }$ denotes the maximum of the total collision rate. The time between collisions (real or null) is then exponentially distributed, with parameter $R_{\text {max }}$, so that collision times can be sampled as

$$
t_{\mathrm{c}}=-\ln (1-U) / R_{\max },
$$

where $U$ is a $[0,1)$ uniform random number. The probability of having a real collision at time $t+t_{\mathrm{c}}$ is now given 
by $\sum_{i} R_{i} / R_{\max }$, using the particle's velocity at the time of collision. Details about our implementation of the null-collision method are given in appendix A.1.

After a collision, electrons are scattered isotropically. This is not realistic, because elastic scattering already becomes anisotropic around $10 \mathrm{eV}$, see for example [35]. However, most electron-neutral cross sections below one $\mathrm{keV}$ are normalized under the assumption of isotropic scattering. For consistency, one should then also use this assumption in the simulation model. From a practical point of view, isotropic scattering is simpler to work with, and obtaining differential electron-neutral cross sections is complicated. This is illustrated at [33]: differential cross sections are only available for argon, based on quantum-mechanical computations [36, 37].

\subsection{Time steps}

There are several time step restrictions in the PIC-MCC simulations. First of all, we impose a CFL-like condition on the particles, so that they move less than half a grid cell: $\Delta t<\frac{1}{2} \Delta x / v_{\max }$. We take $v_{\max }$ as the velocity at 9/10th of the velocity distribution, to reduce fluctuations.

The electric field (see section 2.4) is not always recomputed when the particles are moved, instead a separate time step $\Delta t_{E}$ is used. Each time the field is recomputed, the maximum relative difference $\delta_{E}$ with the previous field is estimated, using 1000 randomly selected samples at particle locations. When $\delta_{E}$ is larger than $\delta_{\max }$, a threshold which we have here set to $7.5 \%, \Delta t_{E}$ is reduced by a factor $\delta_{\max } / \delta_{E}$. When $\delta_{E}<0.5 \delta_{\max }$, $\Delta t_{E}$ is increased by a factor $\min \left[\left(\delta_{\max } / \delta_{E}\right)^{0.1}, 2\right]$.

Although we assume that the plasma in our simulations is weakly ionized, high densities are sometimes produced, for example near the electrode tip. The presence of such regions also imposes a time step restriction, because $\Delta t_{E}$ should be smaller than the dielectric relaxation time $[27,38]$ :

$$
\Delta t_{E}<\varepsilon_{0} /\left(e n_{\mathrm{e}} \mu_{\mathrm{e}}\right)
$$

where $\varepsilon_{0}$ is the dielectric permittivity, $e$ the elementary charge, $n_{\mathrm{e}}$ the electron density and $\mu_{\mathrm{e}}$ the electron mobility. This condition prevents 'over-screening' of an electric field, in which the plasma causes the electric field to change sign and increase in the opposite direction.

To avoid having to use a small time step because of a small high-density region, we artificially limit the electron density to $2.5 \cdot 10^{21} \mathrm{~m}^{-3}$, which corresponds to an ionization degree of $10^{-4}$. Above this density, electrons are gradually converted to negative ions (at most $10 \%$ of the electrons in a cell per update of the electric field). This limits the conductivity of the plasma and prevents oscillations. In most simulations the maximum density is only exceeded in a small region around the electrode tip, and limiting the conductivity there has almost no effect on the discharge evolution.

\subsection{Adaptive mesh refinement for the electric field}

In the electrostatic approximation, the electric field is given by $\mathbf{E}=-\nabla \phi$. Here $\phi$ is the electric potential, obtained by solving Poisson's equation
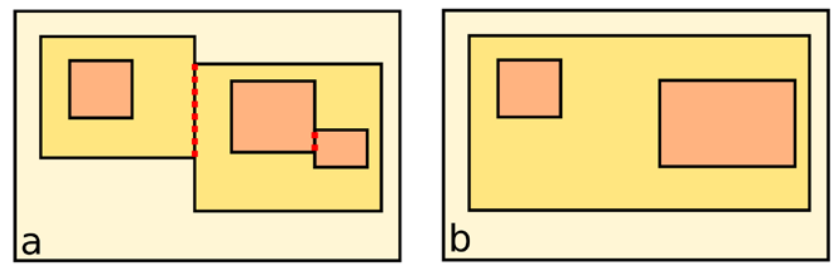

Figure 1. Schematic drawing of a $2 \mathrm{D}$ mesh, to illustrate how refined patches are merged. (a) The regions were refined patches touch are indicated by red dots. (b) The touching regions have been merged into larger rectangles.

$$
\nabla^{2} \phi=-\rho / \varepsilon_{0},
$$

with appropriate boundary conditions; $\rho$ is the charge density. The electrostatic approximation can be used in our simulations because typical electron velocities are much smaller than the speed of light and because induced magnetic fields have negligible effect.

Nanosecond pulsed discharges often have a multiscale nature. At atmospheric pressure, a mesh resolution of a few micrometers is required to resolve their space charge layers, whereas typical discharges measure a few millimeter or more. To speed up simulations, we use a nested grid type of adaptive mesh refinement (AMR), similar to the procedure used in $[39,40]$. An example of such a mesh is shown in figure 3. First, the coordinates at which refinement is needed are collected and grouped into boxes. If two boxes are within two grid points of each other, then they are merged into a single larger box. Each separate box then becomes a new refined grid, with a refinement factor of two. The Dirichlet boundary conditions for the potential on these new grids are interpolated from the parent grid. This procedure is repeated recursively, until there are no more grids to refine.

Fishpack [41] is used to compute the electric potential on each block, after which the electric field is computed by taking central differences. We have experimented with a fineto-coarse correction as described in [42, 43]. Because we observed only modest improvements in our test problems, such a correction was eventually not included.

Because boundary conditions for the potential are interpolated from the parent grid, refined boxes are not allowed to touch each other-this would limit the accuracy at the boundary. For this reason, boxes within two grid points of each other are merged into one larger box. As illustrated in figure 1 , the merging of boxes increases the total number of refined grid points.

The refinement criterion we use depends on the local electric field:

$$
\Delta x<C_{0} / \alpha(E),
$$

where $\alpha(E)$ is the field-dependent ionization coefficient and $C_{0}$ is a constant. The reason for using this criterion is that $1 / \alpha$ is a typical length scale for ionization, so that the space charge layers of a discharge will have a width of a few times $1 / \alpha$. Here we use $C_{0}=2$, which is entirely empirical. This is the highest value that would not significantly change the simulations results. Using a finer mesh increases the computational cost of PIC-MCC simulations in three ways. First, the field 
solver becomes more expensive. Second, more (super-)particles are required, to control the stochastic fluctuations in the finer grid cells, see section 2.6. Third, time steps that depend on the grid becomes smaller, see section 2.3.

We remark that plasma fluid simulations [44] typically require a smaller value of $C_{0} \approx 1$. In such simulations the mesh not only has to resolve the electric field, but also the density profiles of relevant species.

2.4.1. Future improvements. The mesh refinement procedure and field solver described above have two major drawbacks: the field solver is sequential, and the rectangular refinements do not efficiently cover the curved charge layers of discharges. In recent work on 3D plasma fluid simulations, we have resolved these drawbacks by implementing a parallel geometric multigrid solver for octree grids (see [45], chapter 10).

\subsection{Electrode}

When a sparse linear solver is used for Poisson's equation, electrodes can for example be included with the ghost fluid method, as demonstrated in [46]. However, the computational cost of such sparse methods is too high for the 3D simulations presented here. Therefore our field solver is based on Fishpack [41], which can only be used for separable, constantcoefficient elliptic problems on Cartesian grids. Including an electrode can then be done with the capacitance matrix or charge simulation method [23, 47], but these approaches appear unfeasible for the description of an electrode in our 3D simulations, due to the required inversion of the capacitance matrix. We have therefore implemented a custom iterative algorithm, described below.

Our method is based on the linearity and symmetry of Poisson's equation:

$$
\nabla^{2} \phi=-\rho,
$$

where we have omitted $\varepsilon_{0}$ for simplicity. A point charge $\delta\left(\mathbf{r}-\mathbf{r}_{j}\right)$ will affect the potential at $\mathbf{r}_{i}$ by an amount $f_{i, j} \equiv f\left(\mathbf{r}_{i}, \mathbf{r}_{j}\right)$. In free space $f_{i, j} \propto 1 /\left|\mathbf{r}_{i}-\mathbf{r}_{j}\right|$, so that $f_{i, j}=f_{j, i}$. The introduction of Dirichlet boundary conditions does not break this symmetry, as shown in appendix B. More generally, $f_{i, j}=f_{j, i}$ holds for a discrete Poisson equation as long as the discretized Laplace operator $L_{h}$ is symmetric. Then $L_{h}^{-1}$ is also symmetric, from which the property directly follows. In our Poisson solver with Dirichlet boundary conditions we can therefore make use of this symmetry.

To represent an electrode, we generate a large number of points on its surface. For the simulations presented here, these points are spaced by $3 \mu \mathrm{m}$ close to the electrode tip. Away from the tip the spacing is increased to reduce the computational cost. The charges on these points are iteratively updated to bring them close to a potential $V_{0}$, so that they effectively represent an electrode surface. The iterative procedure is described below.

Given $N$ point charges $\delta\left(\mathbf{r}-\mathbf{r}_{i}\right)$ at points $\mathbf{r}_{1}, \mathbf{r}_{2}, \ldots, \mathbf{r}_{N}$, the potential $\phi_{i}$ at each point is computed, and stored as

$$
\lambda_{i}=\phi_{i}=\sum_{j} f_{i, j}
$$

To obtain an average potential $V_{0}$ at the points $\mathbf{r}_{i}$, the point charges are set to $q_{i}=V_{0} / \lambda_{i}$. The potential at each point is then given by

$$
\phi_{i}=\sum_{j} q_{j} f_{i, j}=\sum_{j} V_{0} f_{i, j} / \lambda_{j}
$$

so that the average potential is indeed $V_{0}$ :

$$
\begin{aligned}
\frac{1}{N} \sum_{i} \phi_{i} & =\frac{V_{0}}{N} \sum_{i} \sum_{j} f_{i, j} / \lambda_{j} \\
& =\frac{V_{0}}{N} \sum_{j}\left(\sum_{i} f_{j, i}\right) / \lambda_{j}, \\
& =\frac{V_{0}}{N} \sum_{j} \lambda_{j} / \lambda_{j}=V_{0},
\end{aligned}
$$

where we have used (B.4) and the fact that $f_{i, j}=f_{j, i}$. Although the average potential is now $V_{0}$, the potential at individual points might be far off. Suppose that the differences are $\delta_{i}=V_{0}-\phi_{i}$, then an amount $\delta_{i} / \lambda_{i}$ can be added to each charge $q_{i}$. Writing out the summation as in (B.5) shows that the average potential is then still $V_{0}$. This adjustment of the charges can be repeated, and in all our test cases we observed convergence towards the solution of having $V_{0}$ at each point, up to the discretization error.

With the above update rule for the point charges, the convergence towards $V_{0}$ is rather slow. Fortunately, that is not too much of a problem here. First, in experiments the electrode voltage will also not precisely be $V_{0}$ during a nanosecond pulsed discharge. Second, in the simulations the potential is recomputed at every time step. Because each electrode-iteration starts from the previously determined surface charges, and the potential distribution changes relatively slowly, the slow convergence is less of a problem. After a discharge has formed, the surface charges on the electrode are greatly reduced because of the conductivity of the plasma.

\subsection{Adaptive particle management}

The number of electrons in a typical discharge quickly grows to a value of $10^{8}$ or more. To make particle simulation feasible on modest machines, we have developed an adaptive particle management algorithm [48]. This algorithm uses a $k$-d tree to locate particles that are close in position and velocity. Simulation particles can be merged or split, thereby changing their weights $w$. For merging we use the $\mathbf{v}_{r}$ scheme, in which the velocity is chosen at random from one of the original particles, see [48]. Each particle has a desired weight $w_{\mathrm{d}}$ of

$$
w_{\mathrm{d}}=n_{\mathrm{e}} \Delta x^{3} / N_{\mathrm{ppc}}
$$

where $n_{\mathrm{e}}$ is the local electron density, $\Delta x^{3}$ the volume of the grid cell containing a particle, and $N_{\mathrm{ppc}}$ is the target number of particles per cell, which we set to 32 . The particles for which $w<\frac{2}{3} w_{\mathrm{d}}$ are stored in a $k$-d tree with coordinates $(\mathbf{x}, \lambda|\mathbf{v}|)$, where $\lambda=10^{-12} \mathrm{~s}$, see [48]. Each particle in this tree can then 
be merged with its nearest neighbor. The resulting merged particle gets its position and velocity randomly from one of the original particles.

Particles for which $w>\frac{3}{2} w_{\mathrm{d}}$ can be split into $\left\lceil w_{\mathrm{d}} / w\right\rceil$ new particles. These new particles have the same velocity as the original particle, but their positions are uniformly distributed over a volume $\Delta x^{3} / N_{\mathrm{ppc}}$. This is done to 'smear out' particles moving into a finer grid. In the simulations, merging and splitting is performed each time the total number of particles has grown by a factor of 1.2.

Equation (B.6) gives large weights in the discharge interior, because both $n_{\mathrm{e}}$ and $\Delta x^{3}$ are large there (see section 2.4 for a description of the mesh refinement). In high-field regions where the discharge is actively growing the weights are smaller, because both $n_{\mathrm{e}}$ and $\Delta x^{3}$ are smaller. The use of super-particles with $w>1$ leads to increased density fluctuations, so that one has to be careful when studying a system sensitive to such fluctuations. To investigate to what extent this is a problem in our simulations, simulations for $N_{\mathrm{ppc}}=48$ and $N_{\mathrm{ppc}}=64$ are presented in appendix A.2.

\subsection{Photoionization}

Positive discharges can only grow if there are free electrons ahead of them. These electrons start avalanches that grow towards the discharge, thereby extending it. In nitrogen/ oxygen mixtures, photoionization is often the dominant source of these non-local electrons.

Photoionization can occur when an excited $\mathrm{N}_{2}$ molecule decays via the emission of a UV-photon. If the photon has a wavelength between 98 and $102.5 \mathrm{~nm}$, it can ionize an $\mathrm{O}_{2}$ molecule. Although photoionization is relatively well-studied for $\mathrm{N}_{2} / \mathrm{O}_{2}$ mixtures $[49,50]$, it is not yet clear how to directly generate UV photons from $\mathrm{N}_{2}$ excited states in PIC simulations. We therefore use a stochastic version of Zhelenyak's photoionization model [16], as was done before in [51]. In this model, $\eta$ ionizing photons are (on average) produced per ionization. The factor $\eta$ can be written as $\eta=\eta_{E} \cdot \eta_{\mathrm{q}}$, where $\eta_{E}$ is an efficiency depending on the local electric field $E$ and $\eta_{\mathrm{q}}$ is a quenching-factor. For $\eta_{E}$ we use the data shown in figure 2, taken from [16]. We set the quenching factor $\eta_{\mathrm{q}}$ to

$$
\eta_{\mathrm{q}}=\frac{p_{\mathrm{q}}}{p+p_{\mathrm{q}}}
$$

where $p$ is the pressure and $p_{\mathrm{q}}=30$ mbar the quenching pressure. At $p=p_{\mathrm{q}}$, half the excited states decay due to collisions with other molecules instead of emitting a UV-photon. Equation (B.7) was also taken from Zhelenyak's paper [16], which makes use of data from Teich [52].

If a simulation particle with weight $w$ (representing $w$ physical particles) undergoes an ionization, the number of photons produced is sampled from the Poisson distribution with mean $\eta w$. These photons are always created individually, i.e. a super-particle produces no super-photons. The absorption length of a UV-photon depends on its wavelength. As in [51], we assume wavelengths to be uniformly distributed over the interval from 98 to $102.5 \mathrm{~nm}$. Given a uniform random

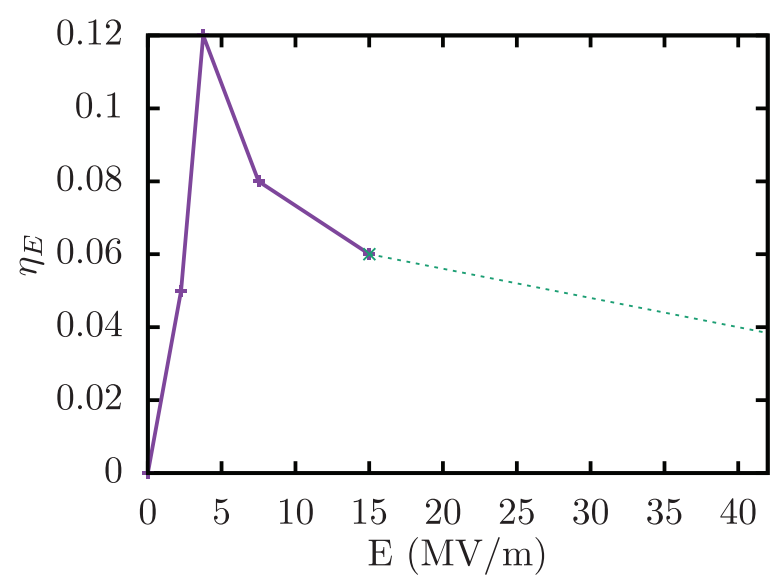

Figure 2. The photoionization coefficient $\eta_{E}$ versus electric field strength. This number indicates how many ionizing photons are produced per electron-impact ionization, in the absence of quenching. The solid curve shows four tabulated points from [16], the dashed curved is an extrapolation of these values that we use for higher fields.

number $R$, the typical absorption length $l$ of a photon is then given by [16]

$$
l=l_{\max }^{R} l_{\min }^{1-R}\left(1 \mathrm{bar} / p_{\mathrm{O}_{2}}\right),
$$

where $l_{\min }=0.67 \mu \mathrm{m}, l_{\max }=0.3 \mathrm{~mm}$ and $p_{\mathrm{O}_{2}}$ is the partial pressure of oxygen (the oxygen fraction times the gas pressure). The average absorption length $\bar{l}_{\gamma}$ resulting from equation (8) is

$$
\bar{l}_{\gamma} \approx 93 \mu \mathrm{m} / p_{\mathrm{O}_{2}}
$$

After the absorption length $l$ has been determined according to equation (8), the actual distance to absorption is sampled from the exponential distribution with mean $l$. The photon gets a random isotropic direction, and at the location of absorption an electron-ion pair is created instantaneously.

We would like to point out some uncertainties within this model:

- We only know the quenching factor (B.7) for air, and assume it to be the same for nitrogen-oxygen mixtures with a lower oxygen content.

- There should be some delay between the generation of an excited state and the emission of a UV-photon. Because we are not aware of good data on this delay, we assume it to be zero in the simulations.

- There are discrepancies between the photoionization rates measured in different experiments, so it is unclear how accurate the above coefficients are, see the discussion in $[49,50]$.

\subsection{Background ionization}

Besides photoionization, there can be other sources of free electrons. Some level of background ionization is always present, mostly in the form of negative ions from which electrons can detach, see for example [21]. Typical densities are $10^{3}-10^{4} \mathrm{~cm}^{-3}$ [49], which is negligible compared to typical 


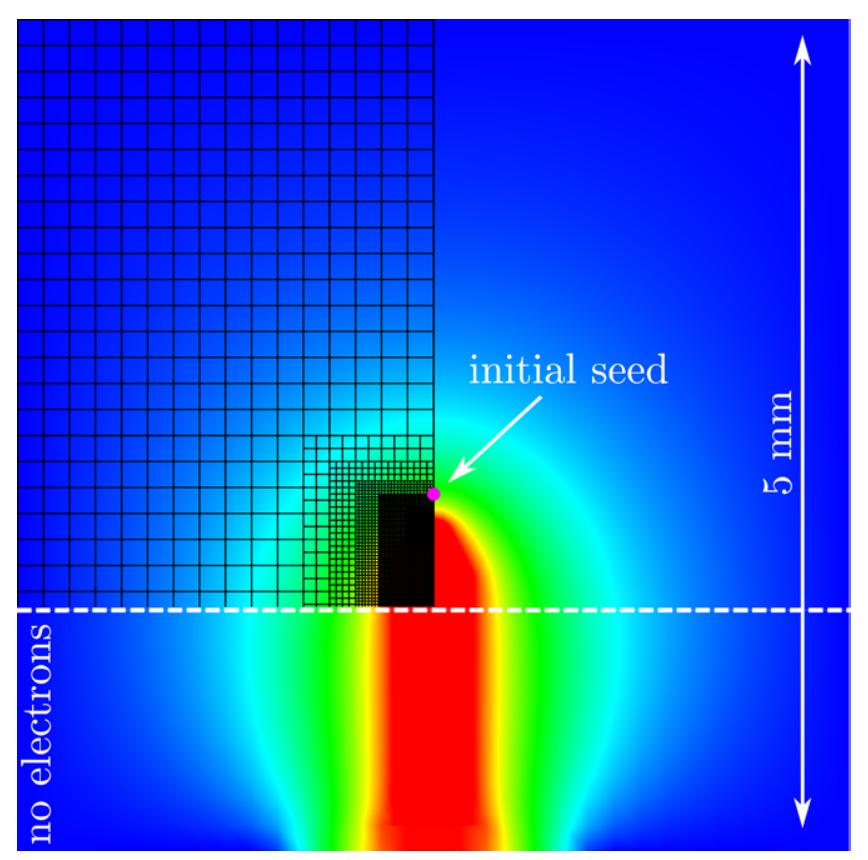

Figure 3. A slice through the 3D computational domain at $t=0$, showing the electrical potential. Red indicates the electrode voltage, blue ground voltage. Part of the numerical mesh at $t=0$ is shown. Electrons are not allowed below the dashed line, to prevent discharges from forming near the bottom boundary where the electrode is embedded. The pink dot indicates the location of the initial seed.

photoionization levels. On the other hand, background ionization can be important to start a discharge, since photoionization only occurs when the discharge has already started [53]. In section 3, we present simulations starting from a background density of $10^{4} \mathrm{~cm}^{-3}$ electrons and ions. Such a density could for example be present in a repetitively pulsed discharge [53].

\subsection{Computational domain}

A slice through the $3 \mathrm{D}$ computational domain is shown in figure 3 , showing the electrical potential at $t=0$. The domain measures $(5.12 \mathrm{~mm})^{3}$, and an electrode with radius $250 \mu \mathrm{m}$ and height $2 \mathrm{~mm}$ is centered at the bottom. This cylindrical electrode ends in a cone (height $500 \mu \mathrm{m}$ ) that transitions into a spherical tip (radius of curvature $125 \mu \mathrm{m}$ ). The side and top walls of the domain are grounded. In the plane with the embedded electrode we use an analytic expression for the potential: it decays linearly to ground potential at three times the electrode radius.

The boundary condition at the bottom can locally create strong electric fields. In experiments, a dielectric material is often used to electrically insulate the electrode from the discharge chamber. We cannot implement this in our field solver, but we still want to prevent discharges from starting there. We therefore do not allow electrons or grid refinement below about $1.5 \mathrm{~mm}$, see figure 3 . We used at most 7 levels of grids, with the coarsest mesh having a resolution of $80 \mu \mathrm{m}$ and the finest a resolution of $1.25 \mu \mathrm{m}$.

\subsection{Parallelization and computing times}

Even with the adaptive particle management described in section 2.6, 3D simulations still require a large number of particles. Therefore, the PIC-MCC code was parallelized using MPI (message passing interface). The simulations presented in section 3 were performed on nodes with 12-16 cores. They took up to $48 \mathrm{~h}$, using at most $6 \cdot 10^{7}$ simulation particles.

\section{Results \& discussion}

The growth of positive discharges depends on the presence of free electrons ahead of them. These electrons propagate towards the discharge, and generate electron avalanches in the high field region around it. If the density of free electrons ahead of the discharge is sufficiently high, individual avalanches cannot be distinguished, and the discharge grows smoothly. When there are few free electrons, the discharge growth is due to individual avalanches, and therefore much more irregular and stochastic.

In nitrogen-oxygen mixtures, photoionization is often the dominant source of non-local free electrons. The photoionization profile around a discharge depends on the oxygen fraction and the gas pressure, as discussed in section 3.1. We have investigated how discharges develop in different nitrogenoxygen mixtures, and how they are affected by the electrode voltage.

Figure 4 shows simulation results for electrode voltages between 3.5 and $5 \mathrm{kV}$, using nitrogen with $0.2 \%$ to $20 \% \mathrm{O}_{2}$ at 1 bar. The computational domain and viewpoint are illustrated in figure 5. For the $5 \mathrm{kV}$ case with $0.2 \%$ and $20 \% \mathrm{O}_{2}$, figure 6 shows cross sections of the electric potential and the electric field. All discharges started from a Gaussian seed placed $130 \mu \mathrm{m}$ above the electrode tip, as indicated in figure 3 . This seed contained $10^{4}$ electron-ion pairs and had a width of $50 \mu \mathrm{m}$. No background ionization was included.

Figure 7 shows simulations at $5 \mathrm{kV}$ for which the ionized seed was replaced by an average density of $10^{4} \mathrm{~cm}^{-3}$ electrons and ions within the whole volume. The positions of the initial electron-ion pairs were generated using random numbers. Results for multiple runs illustrate the variability in the discharges.

In the simulations mentioned above, the electrode tip had a radius of curvature of $r_{\mathrm{c}}=125 \mu \mathrm{m}$, and the electrode voltage was applied instantaneously. Figure 8 shows additional results for electrodes with $r_{\mathrm{c}}=62.5$ and $187.5 \mu \mathrm{m}$, at $5 \mathrm{kV}$ with $20 \%$ oxygen. The effect of including a voltage rise time is briefly discussed in section 3.2.

\subsection{Effect of the oxygen concentration}

The partial pressure of oxygen $p_{\mathrm{O}_{2}}$ affects the photoionization profile around a discharge. The fraction of UV-photons absorbed within a distance $r$ is given by

$$
f(r)=1+\frac{\operatorname{Ei}\left(-r p_{\mathrm{O}_{2}} / l_{\max }\right)-\operatorname{Ei}\left(-r p_{\mathrm{O}_{2}} / l_{\min }\right)}{\ln \left(l_{\min } / l_{\min }\right)},
$$



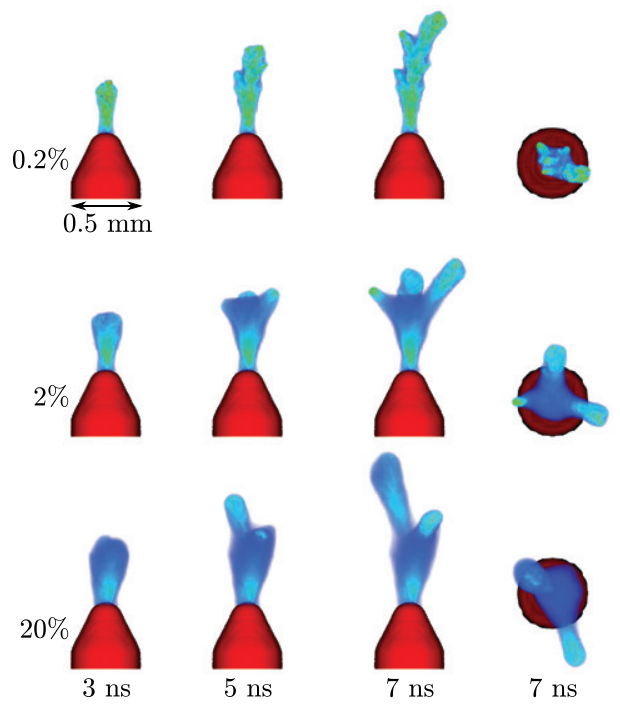
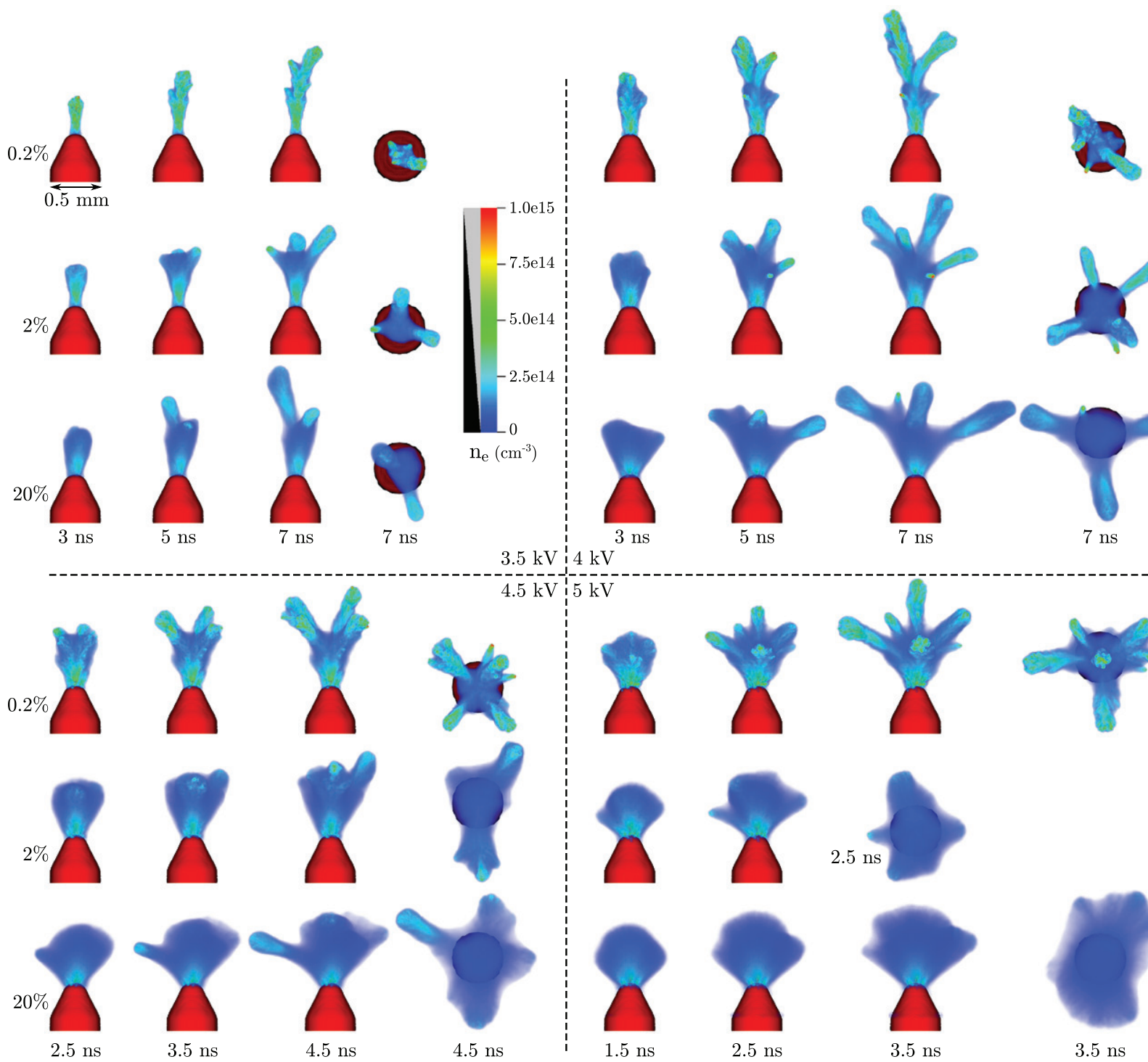

$3.5 \mathrm{kV} 4 \mathrm{kV}$
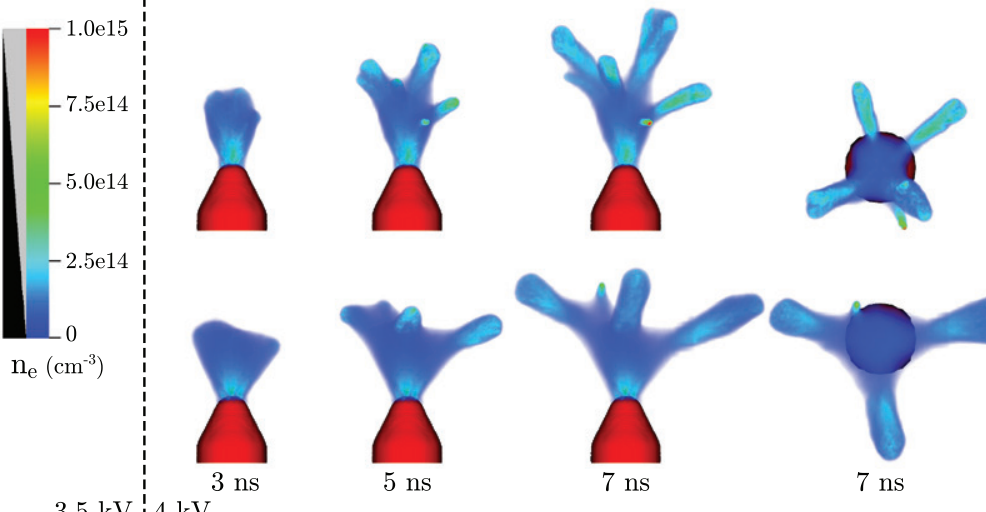

$5 \mathrm{~ns}$
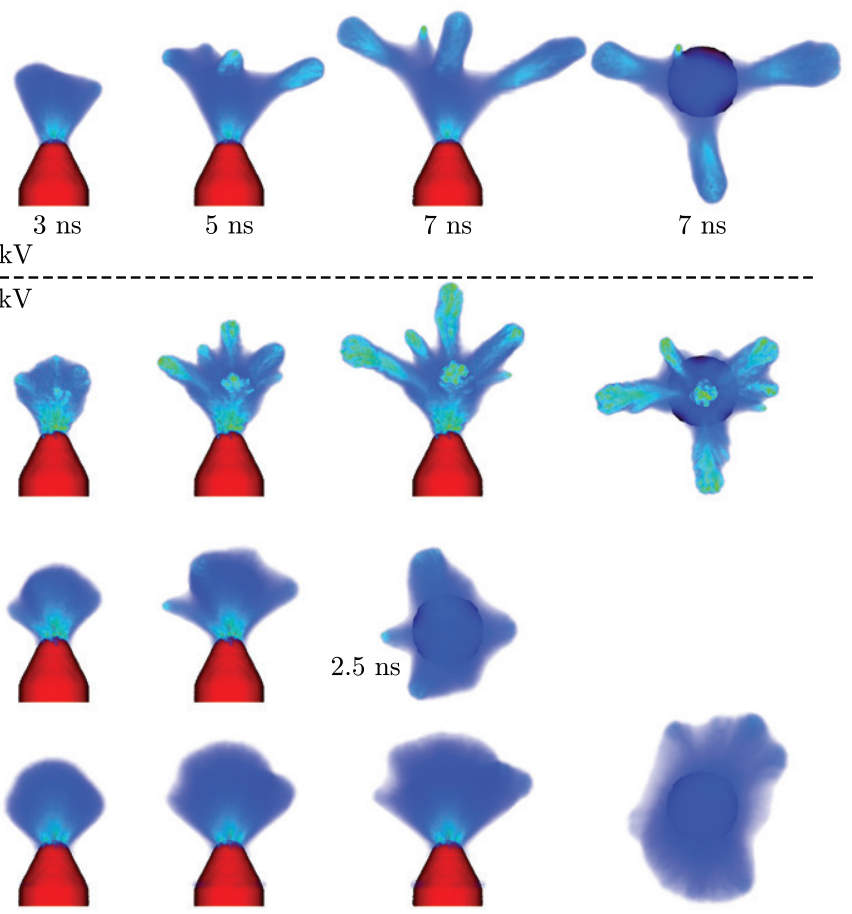

$1.5 \mathrm{~ns}$

$2.5 \mathrm{~ns}$

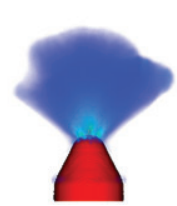

$3.5 \mathrm{~ns}$

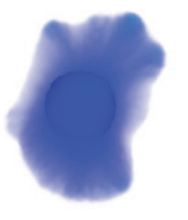

$3.5 \mathrm{~ns}$

Figure 4. Simulation results for electrode voltages between $3.5 \mathrm{kV}$ and $5 \mathrm{kV}$, for nitrogen with $0.2 \%, 2 \%$ and $20 \%$ oxygen at 1 bar. For each simulation, three side views and one top view is given. The $5 \mathrm{kV}$ case with $2 \%$ oxygen did not run up to $3.5 \mathrm{~ns}$, because it required more than $6 \times 10^{7}$ simulation particles. The electron density is visualized with Visit's [54] volume rendering.

in Zhelenyak's model, where $\operatorname{Ei}(x)$ is the exponential integral. In figure $9, f(r)$ is shown for several oxygen concentrations at 1 bar. For values of $r$ smaller than the mean absorption length $\bar{l}_{\gamma}$, see (9), $f(r)$ is approximately proportional to the oxygen concentration. This also follows from the fact that $\bar{l}_{\gamma}$ is itself inversely proportional to $p_{\mathrm{O}_{2}}$. The number of photoionization events in a small volume around the discharge is therefore roughly proportional to $p_{\mathrm{O}_{2}}$.

Figure 4 shows how the discharge evolution is affected by the oxygen concentration. With $20 \%$ oxygen the mean photon absorption length $\bar{l}_{\gamma}$ is about $0.46 \mathrm{~mm}$, for $2 \%$ and $0.2 \%$ it is 10 and 100 times larger, respectively. Because the discharges have a typical size of about a millimeter, the photoionization densities are highest for the cases with $20 \%$ oxygen. These discharges develop more smoothly, they form wider structures and they have a lower degree of ionization. Note that with $0.2 \%$ oxygen the discharges develop quite irregularly, with lots of fine structure, see also figure 10 .

\subsection{The formation of inception clouds}

With $2 \%$ and $20 \%$ oxygen, an almost spherical ionized region forms around the electrode tip, especially at higher voltagessee figure 4. Such inception clouds (also known as 'diffuse discharges', as discussed in the introduction) have been observed experimentally in e.g. [10, 11, 55-57], and their optical emission was compared to simulations in [58]. In a recent experimental study [17] it was found that inception clouds have a typical radius $r_{\text {cloud }}$ of about $0.6 R_{0}$ to $0.9 R_{0}$, where $R_{0}=U / E_{\mathrm{c}}$ is the electrode voltage divided by the critical electric field. We remark that in [17], $r_{\text {cloud }}$ was defined as distance to the electrode tip, and not as half the diameter of the cloud.

The larger an inception cloud gets, the lower the electric field on its surface will be. Assuming that the cloud is equipotential (see figure 6) and nearly spherical with radius $R$, the electric field at its surface can be approximated as $U / R$. The maximal radius of a spherically expanding inception cloud is therefore $R_{0}=U / E_{\mathrm{c}}$. If a cloud reaches this size, growth 
can only continue if a so-called Laplacian instability $[59,60]$ occurs. This means that local electron avalanches [24] create sufficiently large protrusions of the ionization front to locally enhance the electric field. These protrusions will then grow more rapidly due to higher impact ionization rates, while they electrically screen the parts of the front that have stayed behind. In this manner the inception cloud destabilizes into streamer discharges, which penetrate into previously undervolted regions. Besides $R_{0}$, an important length scale for the destabilization process is the typical separation between incoming electron avalanches. If the free electron density ahead of the discharge is $n_{0}$, then the corresponding length scale is $1 / \sqrt[3]{n_{0}}$. This length scale is related to the amount of oxygen and photoionization, as discussed in section 3.1.

We have determined $r_{\text {cloud }}$ for the simulations with $20 \%$ oxygen shown in figure 4 . At $4,4.5$ and $5 \mathrm{kV}$ we get $r_{\text {cloud }} \approx 0.6,0.7$ and $0.8 \mathrm{~mm}$, respectively. For all three voltages, $r_{\text {cloud }}$ corresponds to about $0.5 R_{0}$. The discrepancy with the experimentally determined radii of $0.6 R_{0}$ to $0.9 R_{0}$ could be caused by a number of factors. First of all, the experiments were performed at much higher voltages (tens of $\mathrm{kV}$ ), often at a reduced pressure. This leads to a high degree of ionization around the electrode tip, which generates a relatively high photoionization density around it. At lower pressures there is also less quenching, which increases the amount of photoionization, see equation (B.7). Another cause could be the fact that in experiments the discharges are often repetitively pulsed, which increases the background ionization level.

With 20\% oxygen, the inception clouds shown in figure 4 appear to be slightly larger than with $2 \%$ oxygen, although more simulations would be needed to make this observation statistically significant. With $0.2 \%$ oxygen the discharges quickly break up into streamer channels, so that inception clouds are absent or much smaller. In contrast, in the experiments of Chen et al [17] inception clouds became significantly smaller with $0.01 \%$ oxygen, whereas those at $0.2 \%$ oxygen were about the same size as those at $20 \%$ oxygen. This difference is probably caused by the reasons discussed in the previous paragraph and the fact that the experimental inception clouds were larger, so that absorption lengths for photoionization can also be larger.

To investigate how the size of an inception cloud depends on the initial conditions, we have performed simulations starting from a background density of $10^{4} \mathrm{~cm}^{-3}$ (on average) electrons and ions. Figure 7 shows results for three different runs. In each run, the inception cloud destabilizes at a different moment, but this always happens earlier than in figure 4 , where an initial seed was placed above the electrode tip. With the background density, the initial development takes place where the first major electron avalanches appear, and this early breaking of symmetry prevents the formation of a large inception cloud.

We have also investigated how the radius of curvature of the electrode tip affects the formation of an inception cloud, see figure 8 . With a sharper tip, the inception cloud seems to be more symmetric, leading to a later destabilization into streamers. An explanation for this is that the sharp tip causes a small volume in front of it to become highly ionized, which

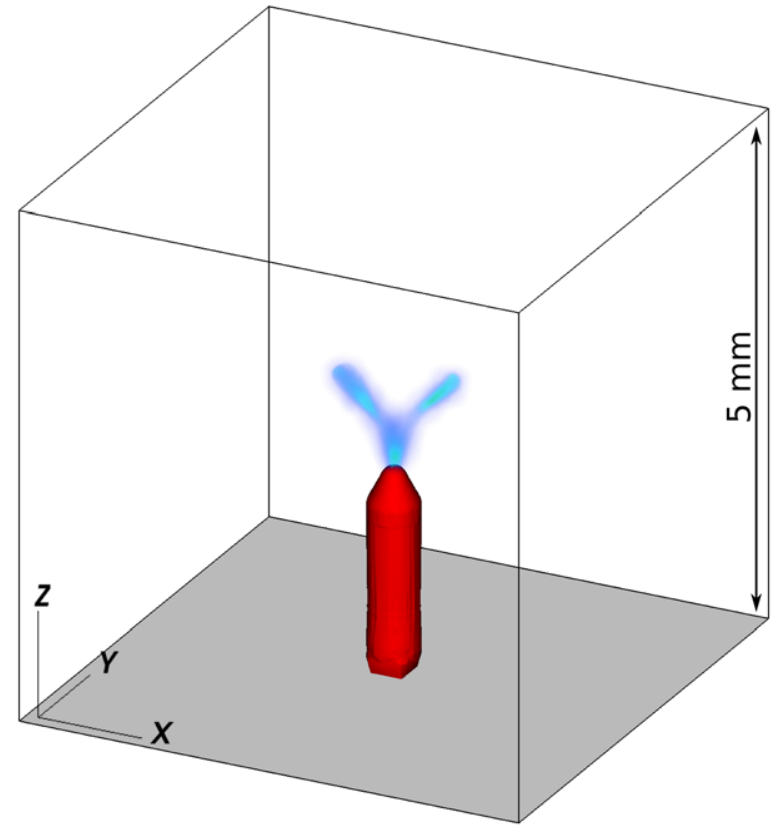

Figure 5. A view of the computational domain with the electrode, used for the simulations presented in section 3. For figures showing the electron density the view is always aligned to the $+y$-axis or to the negative $z$-axis, indicated here.

generates enough photoionization to sustain a smooth and symmetric development afterwards.

In all the simulations, the voltage was applied instantaneously. Including a voltage rise time of a few nanoseconds would cause the discharges to start while the voltage is still rising. This could lead to an earlier destabilization, as was observed in pure nitrogen [57], see also the discussion in [13].

\subsection{Morphology at low oxygen concentrations}

As explained in section 3.1, a low oxygen concentration leads to less photoionization around the discharge. With fewer electron avalanches coming in, the discharge growth is more irregular. Small protrusions enhance the electric field, so that they can grow into streamer channels, see figure 6. This irregular growth shows some qualitative similarities with diffusion limited aggregation [61].

With $0.2 \%$ oxygen, small-scale structure and thin branches are visible in the electron density, see figure 10. Such branches have been observed in several experiments in nitrogen and other pure gases, see for example $[15,19,20,53]$. In these studies, they were referred to as feathers. In $[20,53]$ it was found that the repetition frequency of a pulsed discharge affects the formation of feathers, because it influences the background ionization density.

One question raised in this earlier work was whether feathers are single avalanches [19] or small branches that carry space charge [53]. For our simulations, the answer is clear: feathers are small branches that carry space charge. These branches locally enhance the electric field, but on average the discharge still grows faster in the forward direction. Their growth halts due to a lack of electron avalanches or because the main channel reduces their field enhancement. 

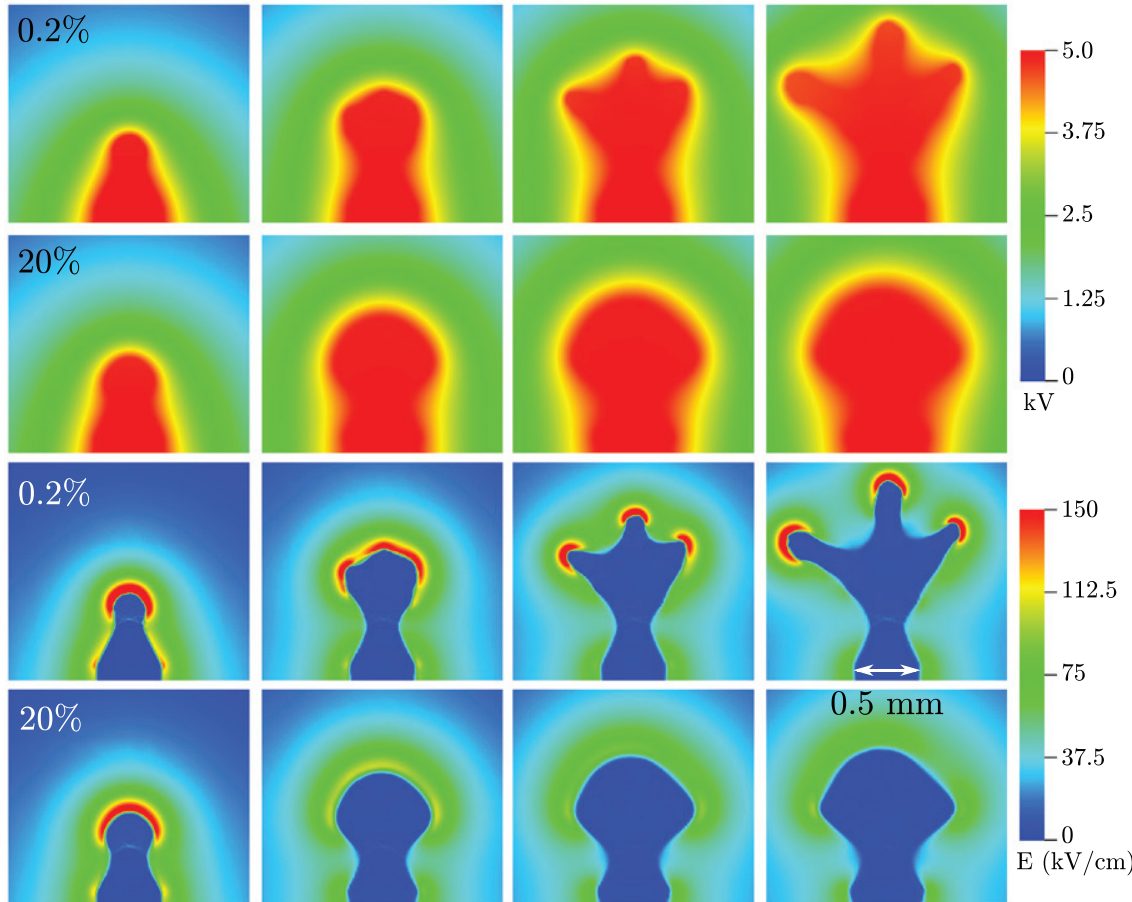

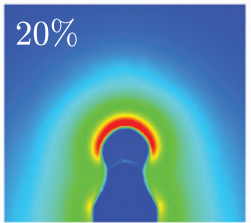

$0.5 \mathrm{~ns}$

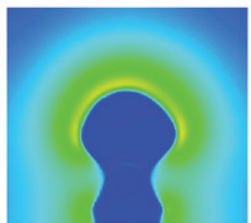

$1.5 \mathrm{~ns}$

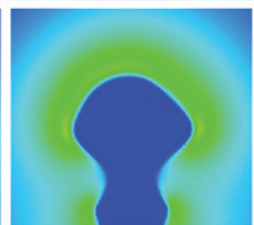

$2.5 \mathrm{~ns}$

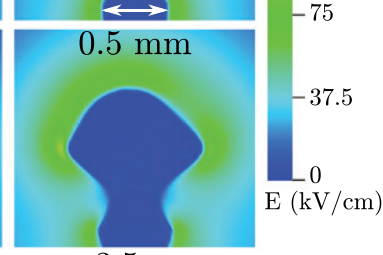

$3.5 \mathrm{~ns}$

Figure 6. Cross sections showing the electric potential and the electric field for simulations in nitrogen with $0.2 \%$ and $20 \%$ oxygen at $5 \mathrm{kV}$, as presented in figure 4.

run 1

$0.2 \%$
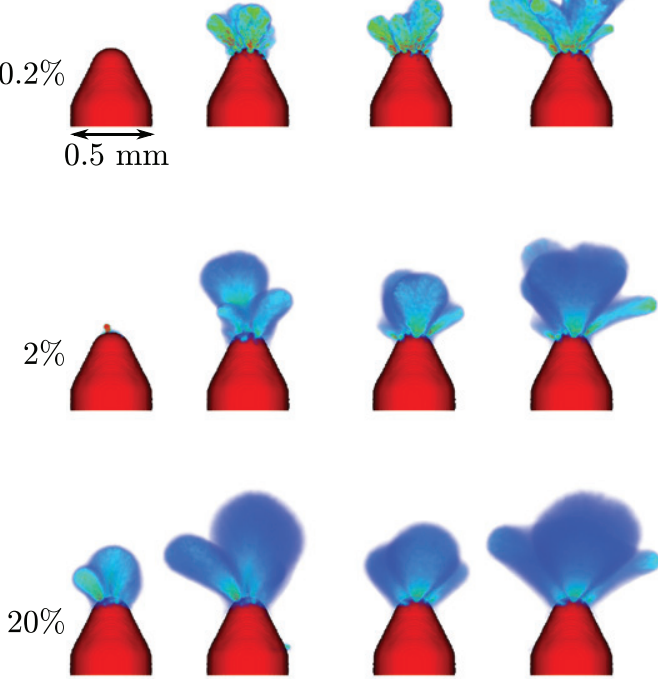

$1.1 \mathrm{~ns}$

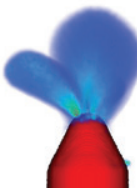

$2.1 \mathrm{~ns}$
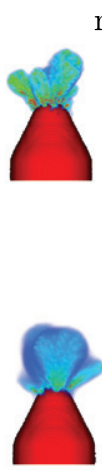

run 2
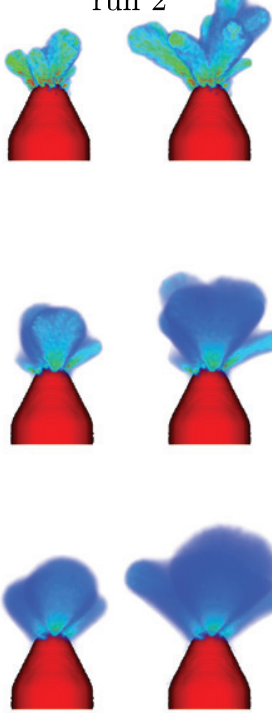

$1.1 \mathrm{~ns}$

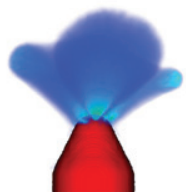

$2.1 \mathrm{~ns}$
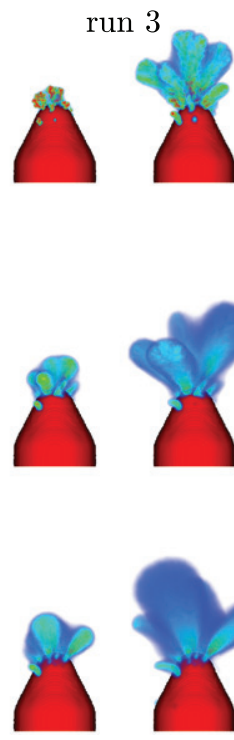

$1.1 \mathrm{~ns}$
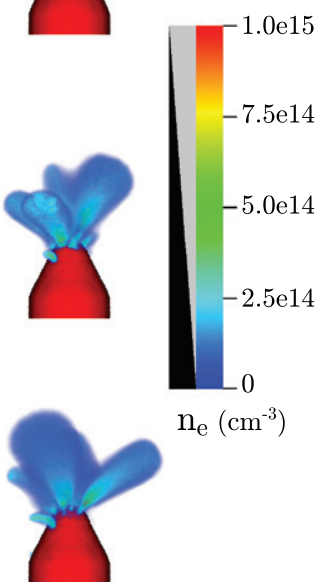

$\mathrm{n}_{\mathrm{e}}\left(\mathrm{cm}^{-3}\right)$

Figure 7. Simulations starting from an average background density of $10^{4} \mathrm{~cm}^{-3}$ electrons and ions, for nitrogen with $0.2 \%, 2 \%$ and $20 \%$ oxygen at $5 \mathrm{kV}$. Run 1, 2 and 3 were performed with different random numbers; they correspond to three different initial electron distributions.

\subsection{Discharge growth velocity}

Figure 4 shows that the discharge growth velocity shows little dependence on the oxygen concentration. This has been observed before for streamer discharges. In [15] streamers propagated with roughly the same velocity when the oxygen concentration was varied over almost six orders of magnitude. In a related simulation study with an axisymmetric model [14], the streamer velocity was also found to be insensitive to the amount of photoionization. We remark that in [14] the photoionization method of [62] was used outside its range of validity. When photon absorption lengths are long compared to the domain size, which is the case in almost pure nitrogen, the approximations of [62] lead to a $1 / r$ decay instead of the correct $1 / r^{2}$ dependence.

With less oxygen the free electron density ahead of a discharge is lower, due to the longer photon absorption length. 

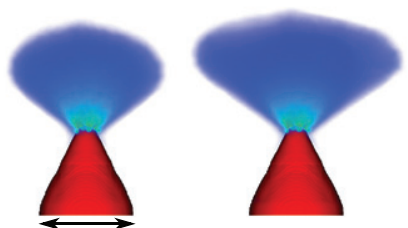

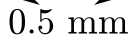
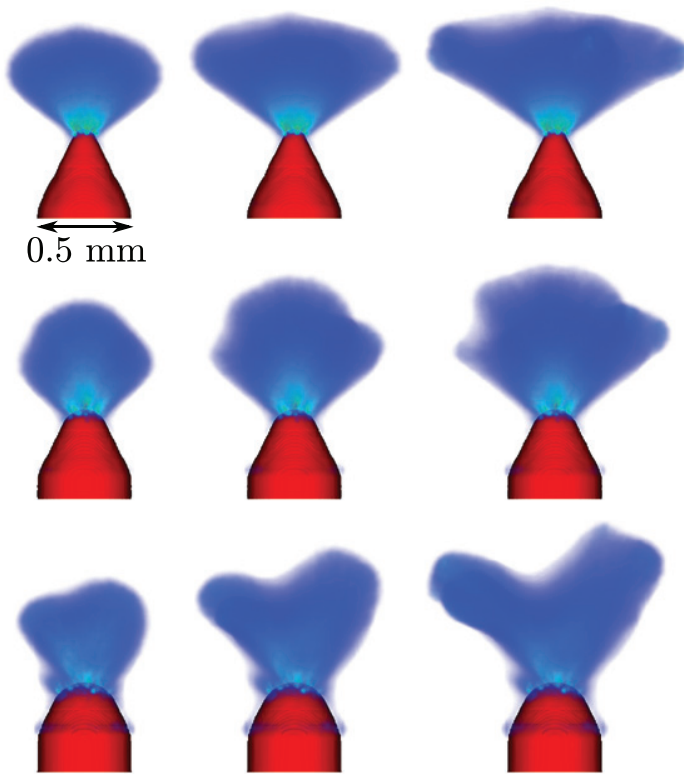

$1.5 \mathrm{~ns}$

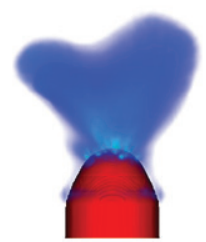

$2.5 \mathrm{~ns}$
$3.5 \mathrm{~ns}$

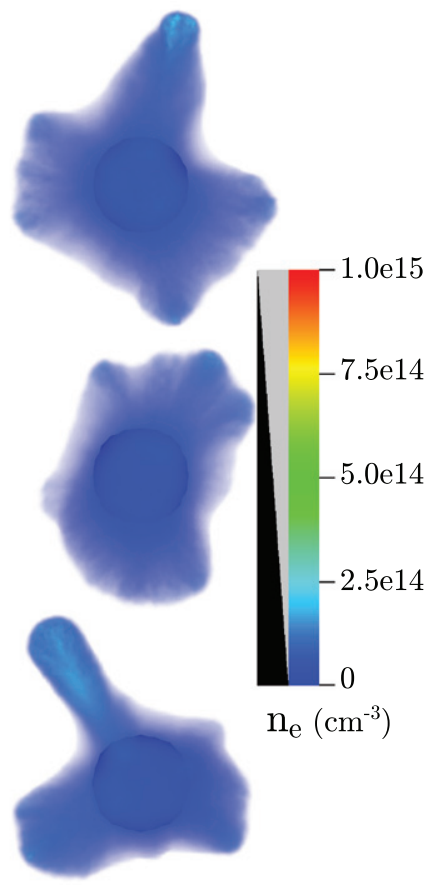

$3.5 \mathrm{~ns}$

Figure 8. Simulation results for a sharper (top, $r_{\mathrm{c}}=62.5 \mu \mathrm{m}$ ) and a blunter (bottom, $r_{\mathrm{c}}=187.5 \mu \mathrm{m}$ ) electrode tip, where $r_{\mathrm{c}}$ is the radius of curvature. The middle row with $r_{\mathrm{c}}=125 \mu \mathrm{m}$ is also shown in figure 4 . The simulations were performed at $5 \mathrm{kV}$ with $20 \% \mathrm{O}_{2}$.

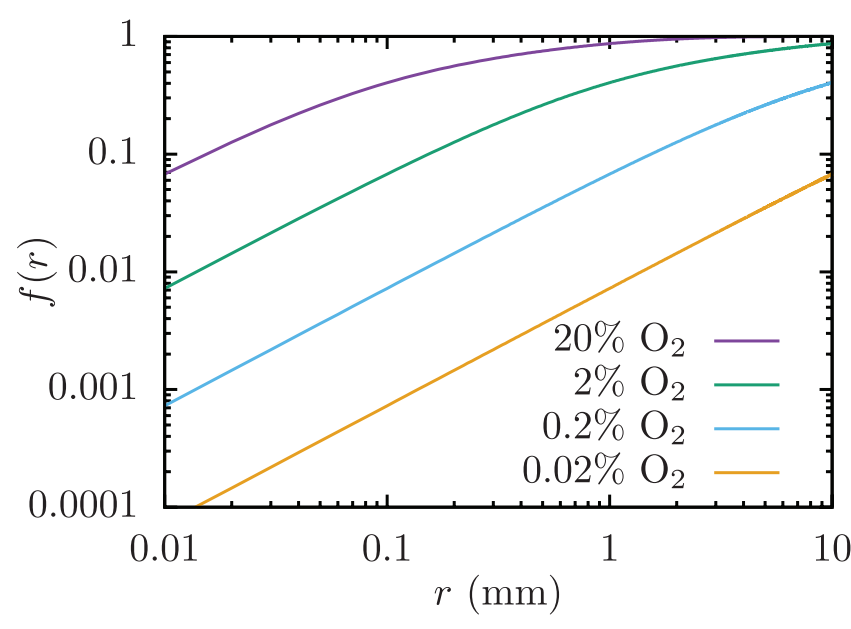

Figure 9. The function $f(r)$ shows the fraction of UV-photons that are absorbed within a distance $r$ after being created, see (10). The curves correspond to different oxygen fractions at 1 bar.

However, this electron density grows approximately exponentially, so a lower starting value has only a minor effect on the time to reach a certain degree of ionization. Furthermore, the amount of oxygen / photoionization will change the morphology of a discharge, as can be seen in figures 4 and 6 . With less oxygen thinner structures emerge, that locally give rise to higher electric fields and therefore higher growth rates.

\section{Conclusions}

This article contains two main contributions: first, a 3D PIC-MCC (particle-in-cell, Monte Carlo collision) with adaptive mesh refinement for its field solver was described, and its

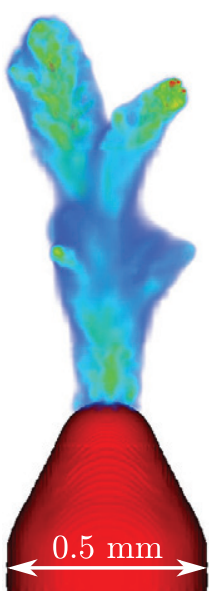

$5 \mathrm{~ns}$

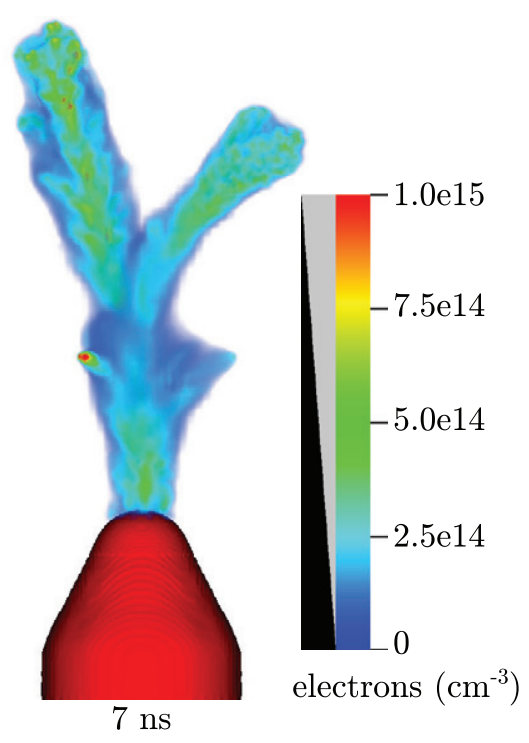

Figure 10. Zoom of the $0.2 \%$ oxygen case at $4 \mathrm{kV}$ of figure 4 . Small-scale protrusions are visible on the sides of the streamer channel.

source code was made available at [30]. Second, this model was used to investigate the inception of nanosecond pulsed discharges. The main advantage of using a particle model in $3 \mathrm{D}$ is that one can observe how stochastic fluctuations affect the discharge. We have performed simulations in a needle-toplane geometry, for voltages between 3.5 and $5 \mathrm{kV}$, and for nitrogen with between $0.2 \%$ and $20 \%$ oxygen.

We found that the discharge velocity was almost independent of the oxygen concentration, in agreement with experimental observations [15]. With $0.2 \%$ oxygen, we observed the formation of thin branches on the discharge, 
which likely correspond to the 'feathers' observed in various experiments $[15,19,53]$. With $2 \%$ or more oxygen and a voltage of 4.5 or $5 \mathrm{kV}$, we observed the formation of an ionized, almost spherical region around the electrode tip. The radius of these observed inception clouds was about a factor two smaller than was found in a recent experimental study [17].

\section{Acknowledgments}

This research is supported by project 10755 of the Dutch Technology Foundation STW, which is part of the Netherlands Organisation for Scientific Research (NWO), and which is partly funded by the Ministry of Economic Affairs.

\section{Appendix A}

\section{A.1. Implementation of the null-collision method}

Our implementation of the null-collision method is described below. First, the $N_{\text {coll }}$ energy-dependent cross sections $\sigma_{i}(\varepsilon)$ are interpolated ${ }^{3}$ to get velocity-dependent collision rates $R_{i}(v)=N \sigma_{i}(v) v$. Then a linear lookup table $T$ is constructed, which at evenly spaced velocities $v_{m}$ stores the cumulative sum of the $R_{i}(v)$, so that $T(m, n) \equiv \sum_{i \leqslant n} R_{i}\left(v_{m}\right)$. The maximum value in the table is stored as $R_{\max }$. The velocities $v_{1}$ to $v_{M}$ are given by $v_{m}=\frac{m-1}{M-1} v_{\max }$, where $v_{\max }$ was set to the velocity of a $1 \mathrm{keV}$ electron and $M$ to $10^{4}$. Because energy varies quadratically with velocity, the table $T$ has a higher resolution at lower energies, where cross sections often show the most complex structure.

To advance a particle at time $t$ to time $t+\delta$, we employ the following procedure:

(i) Store the time remaining $(\delta)$ as $t_{\text {left }}$.

(ii) Sample a collision time $t_{\mathrm{c}}$, see (B.3).

(iii) If $t_{\mathrm{c}} \leqslant t_{\text {left }}$, advance the particle over $t_{\mathrm{c}}$, and subtract $t_{\mathrm{c}}$ from $t_{\text {left }}$. After performing the collision, go back to step (ii).

(iv) If $t_{\mathrm{c}}>t_{\text {left }}$, advance the particle over $t_{\text {left }}{ }^{4}$.

To perform a collision, the table $T$ is interpolated at the particle's velocity $v$, the result of which is stored as list $T_{1}, T_{2}, \ldots, T_{N_{\text {coll }}}$. Then a random rate $R=U R_{\max }$ is drawn, where $U$ is a $[0,1)$ uniform random number. The index of the collision is now the smallest $i$ for which $R \leqslant T_{i}$, and if no such $i$ exists (because $R>T_{N_{\text {coll }}}$ ) there is a null collision. When $N_{\text {coll }}$ is small (e.g. less than 20), a linear search is usually the fastest way to determine $i$, for larger values a binary search becomes more efficient. Our code switches automatically between the two.

Note that the null-collision method can be seen as a type of rejection sampling. When time steps are smaller than typical collision times, the repeated sampling of $t_{\mathrm{c}}$ becomes costly. This can be circumvented by storing a previously calculated collision time until that collision actually occurs. In cases

\footnotetext{
${ }^{3}$ The cross sections should be interpolated as indicated by their authors, in this case linearly in the energy domain.

${ }^{4}$ Note that $t_{\mathrm{c}}$ does not have to be stored because the exponential distribution is memoryless.
}

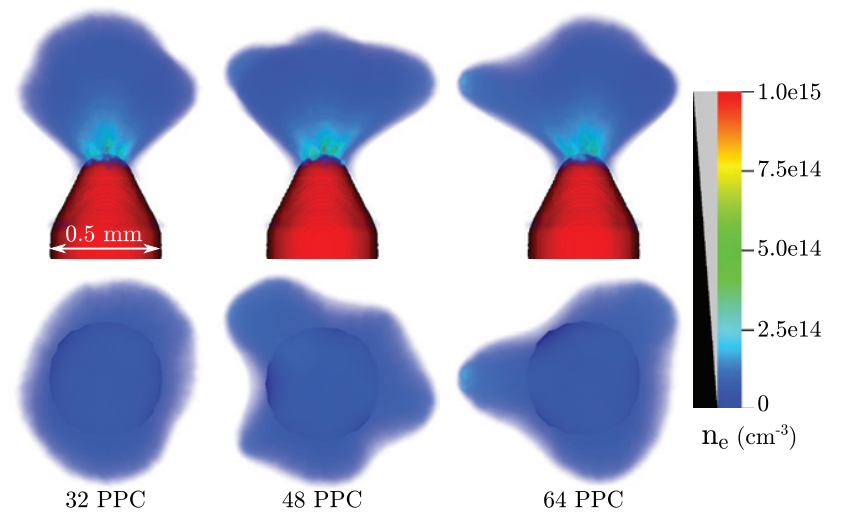

Figure A1. Simulation results at $t=1.9 \mathrm{~ns}$ for three values of $N_{\mathrm{ppc}}$, the number of particles per cell (see section 2.6.) Simulations were performed at $5 \mathrm{kV}$ with $20 \%$ oxygen.

where the gas composition or target density $N$ are not constant, the null collision method can still be used as long as an upper bound for the collision rates is known.

\section{A.2. Varying the number of particles per cell}

In the simulations we use super-particles, see section 2.6. Their weight depends on $N_{\mathrm{ppc}}$, the target number of particles per cell, which was set to 32 . Due to the way we adjust the weights, the number of particles per cell will typically be higher by a factor of about $3 / 2$, so that there are about 50 particles per cell. In regions where there is strong electron impact ionization, the number of particles per cell is even higher, because the merging of particles cannot keep up with their multiplication.

To determine how important the parameter $N_{\mathrm{ppc}}$ is in our model, we have performed simulations for two additional cases: $N_{\mathrm{ppc}}=48$ and $N_{\mathrm{ppc}}=64$, at $5 \mathrm{kV}$ with $20 \% \mathrm{O}_{2}$. The results are shown in figure A1. No apparent differences are visible, which suggests that $N_{\mathrm{ppc}}=32$ is probably high enough for the simulations presented in this paper.

\section{Appendix B. Green's functions for Poisson's equation in bounded domains}

In this appendix, we consider Green's functions $G\left(\mathbf{r}, \mathbf{r}^{\prime}\right)$ for Poisson's equation

$$
\nabla^{2} \phi=-\rho
$$

which are defined as

$$
\nabla^{2} G\left(\mathbf{r}, \mathbf{r}^{\prime}\right)=-\delta\left(\mathbf{r}-\mathbf{r}^{\prime}\right) .
$$

The well-known solution in three-dimensional free space is symmetric in its arguments

$$
G\left(\mathbf{r}, \mathbf{r}^{\prime}\right)=\frac{1}{4 \pi\left|\mathbf{r}-\mathbf{r}^{\prime}\right|}
$$

We demonstrate that this symmetry also holds for different types of boundary conditions, by constructing Green's functions with the help of mirror charges. 
Consider a single Dirichlet boundary condition $\phi=0$ at $x=0$. The Green's function for a point charge at $\mathbf{r}^{\prime}=\left(x^{\prime}, y^{\prime}, z^{\prime}\right)$ then includes an image charge of opposite sign at $\mathbf{r}_{\mathrm{im}}^{\prime}=\left(-x^{\prime}, y^{\prime}, z^{\prime}\right)$, which gives

$$
G\left(\mathbf{r}, \mathbf{r}^{\prime}\right)=\frac{1}{4 \pi}\left(\frac{1}{\left|\mathbf{r}-\mathbf{r}^{\prime}\right|}-\frac{1}{\left|\mathbf{r}-\mathbf{r}_{\mathrm{im}}^{\prime}\right|}\right) .
$$

Note that $G\left(\mathbf{r}, \mathbf{r}^{\prime}\right)$ is symmetric in its arguments and that $G\left(\mathbf{r}, \mathbf{r}^{\prime}\right)=0$ for $x=0$.

If the $\phi=0$ boundary condition is present at $x=0$ and $x=L$, a point charge at $\mathbf{r}^{\prime}=\left(x^{\prime}, y^{\prime}, z^{\prime}\right)$ induces mirror charges of equal sign at the points $\mathbf{r}_{k}^{\prime}=\left(k L+x^{\prime}, y^{\prime}, z^{\prime}\right)$ for all $k= \pm 2, \pm 4, \ldots$, and mirror charges of opposite sign at the points $\left(k L+L-x^{\prime}, y^{\prime}, z^{\prime}\right)$ for $k= \pm 1, \pm 3, \ldots$. The Green's function for this problem is

$$
G\left(\mathbf{r}, \mathbf{r}^{\prime}\right)=\frac{1}{4 \pi} \sum_{k=-\infty}^{\infty} \frac{(-1)^{k}}{\left|\mathbf{r}-\mathbf{r}_{k}^{\prime}\right|},
$$

where $\mathbf{r}_{k}^{\prime}$ is defined as

$$
\mathbf{r}_{k}^{\prime}=\left\{\begin{array}{ll}
\left(k L+x^{\prime}, y^{\prime}, z^{\prime}\right) & k \text { even } \\
\left(k L+L-x^{\prime}, y^{\prime}, z^{\prime}\right) & k \text { odd }
\end{array} .\right.
$$

This Green's function is again symmetric in its arguments and it vanishes at the two boundaries.

For a rectangular box of dimensions $0 \leqslant x \leqslant L_{x}, 0 \leqslant y \leqslant L_{y}$ and $0 \leqslant z \leqslant L_{z}$ with homogeneous Dirichlet boundary conditions, the Green's function can be generalized to

$$
G\left(\mathbf{r}, \mathbf{r}^{\prime}\right)=\frac{1}{4 \pi} \sum_{k, l, m=-\infty}^{\infty} \frac{(-1)^{k+l+m}}{\left|\mathbf{r}-\mathbf{r}_{k l m}^{\prime}\right|},
$$

where $\mathbf{r}_{k l m}^{\prime}$ is the generalization of (B.6). $G\left(\mathbf{r}, \mathbf{r}^{\prime}\right)$ again has the same symmetry and vanishes on the boundaries.

The arguments presented above for homogeneous Dirichlet boundary conditions also hold for non-homogeneous Dirichlet boundaries, because such boundary conditions can be be included by adding a solution to Laplace's equation (i.e. $\rho=0$ ) to $\phi$.

\section{References}

[1] Winands G J J, Yan K, Pemen A J M, Nair S A, Liu Z and Van Heesch E J M 2006 An industrial streamer corona plasma system for gas cleaning IEEE Trans. Plasma Sci. 34 2426-33

[2] van Heesch E J M, Winands G J J and Pemen A J M 2008 Evaluation of pulsed streamer corona experiments to determine the $\mathrm{O}^{*}$ radical yield J. Phys. D: Appl. Phys. 41234015

[3] Pancheshnyi S V, Lacoste D A, Bourdon A and Laux C O 2006 Ignition of propane $\&$ air mixtures by a repetitively pulsed nanosecond discharge IEEE Trans. Plasma Sci. 34 2478-87

[4] Ayan H, Staack D, Fridman G, Gutsol A, Mukhin Y, Starikovskii A, Fridman A and Friedman G 2009 Application of nanosecond-pulsed dielectric barrier discharge for biomedical treatment of topographically nonuniform surfaces J. Phys. D: Appl. Phys. 42125202
[5] Fridman G, Friedman G, Gutsol A, Shekhter A B, Vasilets V N and Fridman A 2008 Applied plasma medicine Plasma Process. Polym. 5 503-33

[6] Winands G J J, Liu Z, Pemen A J M, van Heesch E J M, Yan K and van Veldhuizen E M 2006 Temporal development and chemical efficiency of positive streamers in a large scale wire-plate reactor as a function of voltage waveform parameters J. Phys. D: Appl. Phys. 39 3010-7

[7] Winands G J J, Liu Z, Pemen A J M, van Heesch E J M and Yan K 2008 Analysis of streamer properties in air as function of pulse and reactor parameters by ICCD photography J. Phys. D: Appl. Phys. 41234001

[8] Huiskamp T, van Heesch E J M and Pemen A J M 2015 Final implementation of a subnanosecond rise time, variable pulse duration, variable amplitude, repetitive, high-voltage pulse source IEEE Trans. Plasma Sci. 43 444-51

[9] Huiskamp T 2015 Nanosecond pulsed power technology for transient plasma generation $P h D$ Thesis Technische Universiteit Eindhoven http://repository.tue.nl/798746

[10] Tardiveau P, Moreau N, Bentaleb S, Postel C and Pasquiers S 2009 Diffuse mode and diffuse-to-filamentary transition in a high pressure nanosecond scale corona discharge under high voltage J. Phys. D: Appl. Phys. 42175202

[11] Shao T, Zhang C, Niu Z, Yan P, Tarasenko V F, Baksht E Kh, Burahenko A G and Shut'ko Y V 2011 Diffuse discharge, runaway electron, and $\mathrm{x}$-ray in atmospheric pressure air in an inhomogeneous electrical field in repetitive pulsed modes Appl. Phys. Lett. 98021503

[12] Briels T M P, van Veldhuizen E M and Ebert U 2008 Time resolved measurements of streamer inception in air IEEE Trans. Plasma Sci. 36 908-9

[13] Briels T M P, Kos J, van Veldhuizen E M and Ebert U 2006 Circuit dependence of the diameter of pulsed positive streamers in air J. Phys. D: Appl. Phys. 39 5201-10

[14] Wormeester G, Pancheshnyi S, Luque A, Nijdam S and Ebert U 2010 Probing photo-ionization: simulations of positive streamers in varying $\mathrm{N}_{2}: \mathrm{O}_{2}$-mixtures $J$. Phys. $D$ : Appl. Phys. 43505201

[15] Nijdam S, van de Wetering F M J H, Blanc R, van Veldhuizen E M and Ebert U 2010 Probing photoionization: experiments on positive streamers in pure gases and mixtures J. Phys. D: Appl. Phys. 43145204

[16] Zheleznyak M B, Mnatsakanian A K and Sizykh S V 1982 Photoionization of nitrogen and oxygen mixtures by radiation from a gas discharge Teplofiz. Vys. Temp. 20 423-8

[17] Chen S, Heijmans L C J, Zeng R, Nijdam S and Ebert U 2015 Nanosecond repetitively pulsed discharges in $\mathrm{N}_{2}-\mathrm{O}_{2}$ mixtures: inception cloud and streamer emergence J. Phys. D: Appl. Phys. 48175201

[18] van Veldhuizen E M and Rutgers W R 2003 Inception behaviour of pulsed positive corona in several gases $J$. Phys. D: Appl. Phys. 36 2692-6

[19] Wormeester G, Nijdam S and Ebert U 2011 Feather-like structures in positive streamers interpreted as electron avalanches Japan J. Appl. Phys. 50 08JA01

[20] Heijmans L C J, Clevis T T J, Nijdam S, van Veldhuizen E M and Ebert U 2015 Streamer knotwilg branching: sudden transition in morphology of positive streamers in highpurity nitrogen J. Phys. D: Appl. Phys. 48355202

[21] Sun A, Teunissen J and Ebert U 2014 The inception of pulsed discharges in air: simulations in background fields above and below breakdown J. Phys. D: Appl. Phys. 47445205

[22] Birdsall C K 1991 Particle-in-cell charged-particle simulations, plus Monte Carlo collisions with neutral atoms, PIC-MCC IEEE Trans. Plasma Sci. 19 65-85

[23] Hockney R W and Eastwood J W 1988 Computer Simulation Using Particles (Bristol: IOP Publishing)

[24] Luque A and Ebert U 2011 Electron density fluctuations accelerate the branching of positive streamer discharges in air Phys. Rev. E 84046411 
[25] Li C, Teunissen J, Nool M, Hundsdorfer W and Ebert U 2012 A comparison of 3D particle, fluid and hybrid simulations for negative streamers Plasma Sources Sci. Technol. 21055019

[26] Nijdam S, Takahashi E, Teunissen J and Ebert U 2014 Streamer discharges can move perpendicularly to the electric field New J. Phys. 16103038

[27] Teunissen J, Sun A and Ebert U 2014 A time scale for electrical screening in pulsed gas discharges $J$. Phys. D: Appl. Phys. 47365203

[28] Sun A B, Teunissen J and Ebert U 2013 Why isolated streamer discharges hardly exist above the breakdown field in atmospheric air Geophys. Res. Lett. 40 2417-22

[29] Sun A, Teunissen J and Ebert U 2014 3-D particle modeling of positive streamer inception from a needle electrode in supercritical nitrogen IEEE Trans. Plasma Sci. 42 2416-7

[30] Multiscale Dynamics group. CWI multiscale dynamics webpage http://cwimd.nl (accessed May 2016)

[31] Verlet L 1967 Computer 'experiments' on classical fluids. I. Thermodynamical properties of Lennard-Jones molecules Phys. Rev. 159 98-103

[32] Pitchford L C and Boeuf J P 2013 Siglo database, www.lxcat. net (retrieved April 2013)

[33] LXCat Team 2015 The LXCat project http://lxcat.net [online; accessed 26 July 2015]

[34] Koura K 1986 Null-collision technique in the direct-simulation Monte Carlo method Phys. Fluids 293509

[35] Okhrimovskyy A, Bogaerts A and Gijbels R 2002 Electron anisotropic scattering in gases: a formula for Monte Carlo simulations Phys. Rev. E 65037402

[36] Zatsarinny O and Bartschat K 2004 B-spline Breit-Pauli R-matrix calculations for electron collisions with argon atoms J. Phys. B: At. Mol. Opt. Phys. 37 4693-706

[37] Allan M, Zatsarinny O and Bartschat K 2006 Near-threshold absolute angle-differential cross sections for electronimpact excitation of argon and xenon Phys. Rev. A 74030701

[38] Barnes M S, Cotler T J and Elta M E 1987 Large-signal timedomain modeling of low-pressure RF glow discharges $J$. Appl. Phys. 6181

[39] Wackers J 2005 A nested-grid direct poisson solver for concentrated source terms J. Comput. Appl. Math. $1801-12$

[40] Montijn C, Hundsdorfer W and Ebert U 2006 An adaptive grid refinement strategy for the simulation of negative streamers J. Comput. Phys. 219 801-35

[41] Adams J, Swarztrauber P and Sweet R 2011 Fishpack90 http:// cisl.ucar.edu/css/software/fishpack90 [online; accessed 2011]

[42] Ricker P M 2008 A direct multigrid poisson solver for oct-tree adaptive meshes Astrophys. J. Suppl. Ser. 176 293-300

[43] Huang J and Greengard L 1999 A fast direct solver for elliptic partial differential equations on adaptively refined meshes SIAM J. Sci. Comput. 21 1551-66

[44] Luque A and Ebert U 2012 Density models for streamer discharges: beyond cylindrical symmetry and homogeneous media J. Comput. Phys. 231 904-18
[45] Teunissen J 2015 3D simulations and analysis of pulsed discharges PhD Thesis Technische Universiteit Eindhoven http://repository.tue.nl/801516

[46] Celestin S, Bonaventura Z, Zeghondy B, Bourdon A and Ségur P 2009 The use of the ghost fluid method for Poisson's equation to simulate streamer propagation in point-to-plane and point-to-point geometries J. Phys. D: Appl. Phys. 42065203

[47] Malik N H 1989 A review of the charge simulation method and its applications IEEE Trans. Electr. Insul. 24 3-20

[48] Teunissen J and Ebert U 2014 Controlling the weights of simulation particles: adaptive particle management using k-d trees J. Comput. Phys. 259 318-30

[49] Pancheshnyi S 2005 Role of electronegative gas admixtures in streamer start, propagation and branching phenomena Plasma Sources Sci. Technol. 14 645-53

[50] Pancheshnyi S 2014 Photoionization produced by low-current discharges in $\mathrm{O}_{2}$ air, $\mathrm{N}_{2}$ and $\mathrm{CO}_{2}$ Plasma Sources Sci. Technol. 24015023

[51] Chanrion O and Neubert T 2008 A PIC-MCC code for simulation of streamer propagation in air J. Comput. Phys. $2277222-45$

[52] Teich T H 1967 Emission gasionisierender strahlung aus elektronenlawinen Z. Phys. 199 378-94

[53] Nijdam S, Wormeester G, van Veldhuizen E M and Ebert U 2011 Probing background ionization: positive streamers with varying pulse repetition rate and with a radioactive admixture J. Phys. D: Appl. Phys. 44455201

[54] Childs H et al 2012 VisIt: an end-user tool for visualizing and analyzing very large data High Performance VisualizationEnabling Extreme-Scale Scientific Insight (London: Chapman and Hall) pp 357-72

[55] Briels T M P, van Veldhuizen E M and Ebert U 2008 Positive streamers in air and nitrogen of varying density: experiments on similarity laws J. Phys. D: Appl. Phys. 41234008

[56] Nijdam S, Miermans K, van Veldhuizen E M and Ebert U 2011 A peculiar streamer morphology created by a complex voltage pulse IEEE Trans. Plasma Sci. 3922162217

[57] Clevis T T J, Nijdam S and Ebert U 2012 Inception and propagation of positive streamers in high-purity nitrogen: effects of the voltage rise rate J. Phys. D: Appl. Phys. 46045202

[58] Pechereau F, Le Delliou P, Jansky J, Tardiveau P, Pasquiers S and Bourdon A 2014 Large conical discharge structure of an air discharge at atmospheric pressure in a point-to-plane geometry IEEE Trans. Plasma Sci. 42 2346-7

[59] Arrayás M, Ebert U and Hundsdorfer W 2002 Spontaneous branching of anode-directed streamers between planar electrodes Phys. Rev. Lett. 88174502

[60] Ebert U et al 2010 Multiple scales in streamer discharges, with an emphasis on moving boundary approximations Nonlinearity 24 C1-26

[61] Niemeyer L, Pietronero L and Wiesmann H 1984 Fractal dimension of dielectric breakdown Phys. Rev. Lett. 52 1033-6

[62] Luque A, Ebert U, Montijn C and Hundsdorfer W 2007 Photoionization in negative streamers: fast computations and two propagation modes Appl. Phys. Lett. 90081501 